\title{
RESEARCH ON MICROPHYSICAL PROPERTIES OF A VARIETY OF NONSPHERICAL AEROSOL PARTICLES
}

\author{
Yapeng $\mathrm{Liu}^{1}$, Hu Zhao ${ }^{2, *}$, Hailun Zhang ${ }^{2}$, Xingkai Wang ${ }^{1}$, Chang Shu ${ }^{1}$ \\ ${ }^{1}$ School of computer science and engineering, North MinZu University, Yinchuan, 750021, China - (982843635, 1395585521, \\ 410513982)@qq.com \\ ${ }^{2}$ School of Electrical and Information Engineering, North MinZu University, Yinchuan, 750021, China - zhaoh_1@yeah.net, \\ 809361497@qq.com
}

Commission III, WG III/8

KEY WORDS: Aerosol, Nonspherical, Scattering Properties, T-matrix, Atmosphere

\begin{abstract}
:
In order to study the environment or climate of an area, it is necessary to understand the composition of atmospheric aerosol particles, as well as microphysical properties, such as extinction cross section, scattering cross section, polarization degree, etc. For a long time, when calculating the microphysical properties of atmospheric aerosol particles, the aerosol particles are always be considered as spheres. Mie theory has been used to calculate the scattering properties of spherical particles with high accuracy. However, in reality, aerosol particles are not only spherical, they have complex composition and different shapes. The influence of non-spherical aerosol particles on atmospheric radiation, scattering and absorption cannot be ignored. Therefore, it is necessary to fully understand the micro-physical characteristics of non-spherical aerosol particles for fully understand the real atmospheric environment. Until now, T-Matrix method is one of the most effective and extensive methods to study the light scattering characteristics of non-spherical rotationally symmetric aerosol particles. In this paper, the non-spherical aerosol particles extinction section, scattering cross section, the absorption cross section are calculated using T-Matrix method. The extinction, scattering, and the absorption properties are calculated with variety of different types aerosol particles, and compared with the properties calculated by Mie scattering theory. It lays a foundation for more accurate simulation of the microphysical properties of aerosol particles in real atmosphere.
\end{abstract}

\section{INTRODUCTION}

Generally, in order to simplify the calculation, researchers regard aerosol particles in the atmosphere as spheres in the study of aerosol scattering characteristics. However in the actual atmospheric environment, it is an aggregation of many spherical and non-spherical irregular aerosol particles. It is going to make a lot of errors regard aerosol particles as spheres(Mishchenko et al. 2002), considering the many kind of non-spherical particle shapes adequately can make the calculation result suitable to the actual situation. In the calculation of scattering characteristics of spherical particles, the classical Lorenz-Mie scattering theory is simple and practical. It can calculate the scattering characteristics of uniform spherical particles accurately. But it cannot simulate the actual atmosphere conditions accurately (Huber and Frost, 1998). Hoyningen-Huene(Hoyningen-Huene et al. 1997)proved that the scattering energy calculated by Lorenz-Mie theory in non-spherical particles has very large error. In the calculation of non-spherical particles, the Lorenz-Mie theory has some limitations. In order to calculate the scattering characteristics of non-spherical particles conveniently, at present, it is mainly divided into integral method and difference method(Hovenier et al. 2000). For example, there are Discrete Dipole Approximation(DDA), Finite-difference Time-domain(FDTD), Finite Element Method(FEM), Separation of Variable Method (SVM), T-Matrix method,et al(Farafonov et al. 2005).

Discrete Dipole Approximation (DDA) is a dipole equivalent scattering. The arrays of small dipoles can describe the simulated non-spherical aerosol particles(Purcell et al. 1973). It converts the study of actual targets to the study of small dipoles. The advantage of this method is that it can automatically satisfy the infinite radiation condition, and can be used for any uniform, isotropic and rotating light scattering(Draine, 1988). However, the main disadvantage of this approach is that it is not suitable for the condition of very large size parameter or very large refractive index. It can only get low computational accuracy. When the particle size parameter increases, the improvement of CPU performance is not obvious, and the computational complexity is high and the memory consumption is large.

Finite-difference Time-domain (FDTD) is a set of time-domain advance formulas obtained by differential discretization of Maxwell's curl equations, then the electromagnetic scattering problem is calculated(Umashankar and Taflove, 1982), in short, it's a direct time domain solution. FDTD first calculates the electromagnetic field value in the near region, and then obtains the far field value through the near field, and then deduces the scattering field value outside the calculation domain of the whole FDTD(Yang and Liou, 1996). The computation of FDTD can only be carried out in a limited region, so an important aspect is the effective construction of absorbing boundary conditions(Schneider, 2004, Mur, 1981).Poor performance or inappropriate absorption boundary conditions may require a large free space between the model scattering and the boundary, leading to a large amount of computation, high memory consumption and repeated calculation.

* Corresponding author: Hu Zhao

E-mail: zhaoh_1@yeah.net 
Finite Element Method(FEM)(Jin, 2002) shows that the solution domain is composed of many small interconnected subdomains, assuming a suitable approximate solution for each element, and then deducing and solving the overall solution of this domain. FEM has the advantage that it is very simple to model arbitrary shapes and non-uniform particles. However, since FEM has to be calculated for a specific region, it greatly increases the running time of the algorithm, the more the element, the greater the memory requirement(Choi et al. 1996). Moreover, FEM is suitable for small particle size, and it is not easy to obtain high numerical accuracy.

The essence of the Separation of Variable Method (SVM) is to solve the vector Helmholtz equation(Rother, 1998) of timeharmonic electric field. Its calculation accuracy is very high, and it is general and easy to match the boundary conditions(Schmidt et al. 2003). Therefore, it can be used to simulate the scattering characteristics of ellipsoidal particles with large axial ratio. However, it can only be used when the size parameter is small, which is not suitable for the case of larger particle size and larger refractive index in the environment.

However, until now, T-matrix method(Spurr et al. 2012) is still one of the most effective and extensive methods to study the light scattering characteristics of non-spherical rotationally symmetric particles. The T-matrix method is a technique for calculating electromagnetic scattering of a single uniformly rotating non-spherical particle based on the extended boundary condition principle(Wielaard et al. 1997).The T-matrix method was first proposed by waterman(Mishchenko and Travis, 1994), The T-matrix scattering theory method is to express the expansion coefficient of the vector spherical harmonic function of the scattering field with the expansion coefficient of the vector spherical harmonic function of the incident field(Waterman, 1965), and the connecting is the T-matrix. The basic feature of $\mathrm{T}$ matrix is that the scattering characteristics are only related to refractive index, size and shape, and are not restricted by other factors, so they are completely independent of incident and scattering field. Therefore, the T-matrix only needs to be calculated once, and then it is completely determined. So it can be accurately used to calculate the light incidence and scattering in any random direction.

T-matrix method is suitable for random directional uniform and symmetric particles. In this paper, the light scattering characteristics of spherical and non-spherical aerosol particles calculated by T-matrix are compared with the results calculated by Mie scattering theory, in order to verify the calculation accuracy of t-matrix and analyze the error of the results. This paper lays a foundation for calculating the properties of nonspherical aerosol particles.

\section{T-MATRIX METHOD}

T-Matrix method is based on the Maxwell equation method to calculate the scattering characteristics of spherical particles. The particles in the frame of reference to T-Matrix of a single homogeneous scattering standard scheme called extended boundary condition method (EBCM). A transfer Matrix between incident field and scattering field coefficient obtained using the boundary conditions of generalized analytic coefficient. It only related to the inherent physical characteristics of particles.

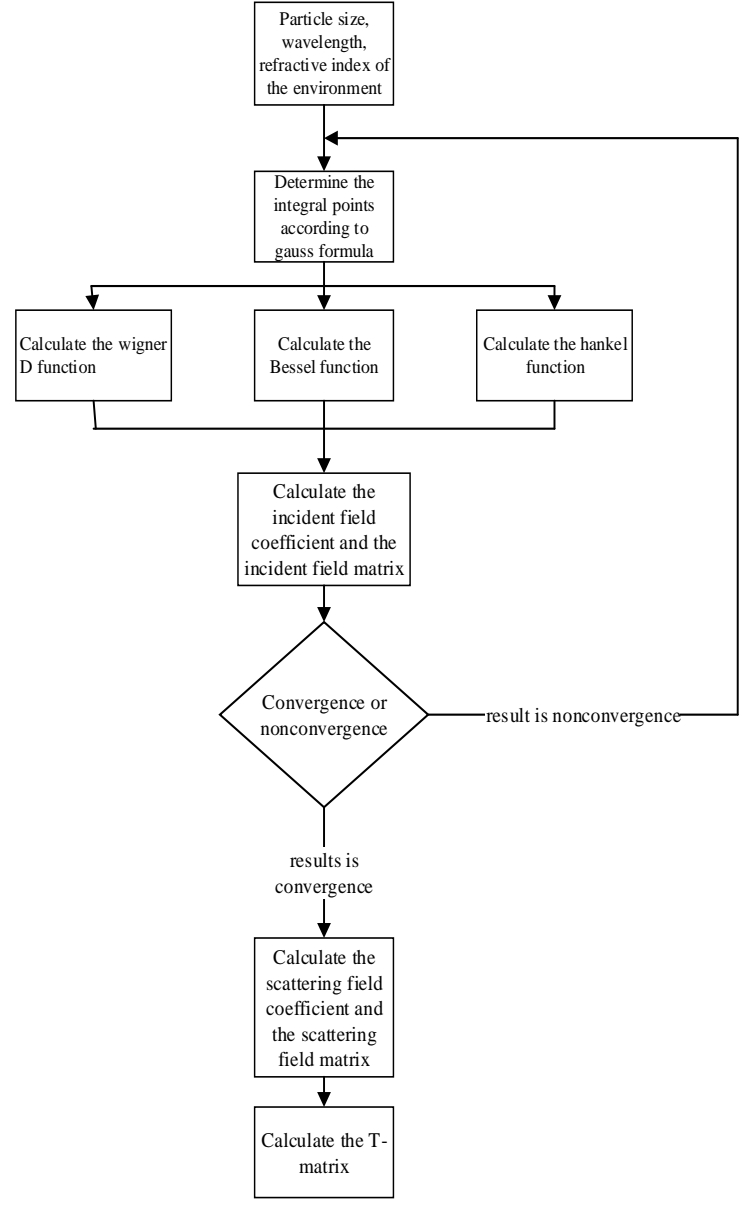

Figure 1. The process of calculating the T-Matrix

Figure 1 is the process of calculating the T-matrix of the incident field and scattering field. The key to the calculation is whether the incident field coefficient converges.

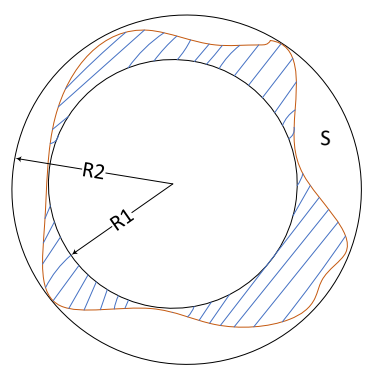

Figure 2. Diagram about non-spherical particle analysis

Figure 2 is the scattering cross section of general aerosol particles bounded by the closed surface $\mathrm{S}$. R2 is the minimum sphere radius of the aerosol particles centered at the origin of the coordinate system. R1 is the radius of the concentric sphere contact.

For electromagnetic scattering of any fixed and uniform object, under the irradiation of the incident field, the surface of nonspherical aerosol particles will generate an electric current, which stimulates the scattering field, the internal electric field and scattering electric field（错误!未找到引用源。, 错误!未 找到引用源。and错误!未找到引用源。) (Park and Minh, 
2012) are represented by vector spherical wave functions 错误! 未找到引用源。 and 错误!未找到引用源。 respectively:

$$
\begin{aligned}
E_{i n c}(R) & =\sum_{n=1}^{n_{\max }} \sum_{m=-n}^{n}\left[a_{m n} R g M_{m n}(k R)+b_{m n} R g N_{m n}(k R)\right] \\
E_{p a r}(R) & =\sum_{n=1}^{n_{\max }} \sum_{m=-n}^{n}\left[c_{m n} R g M_{m n}\left(m_{c} k R\right)+d_{n n} R g N_{m n}\left(m_{c} k R\right)\right] \\
E_{s c a}(R) & =\sum_{n=1}^{n_{\max }} \sum_{m=-n}^{n}\left[p_{m n} M_{m n}(k R)+q_{m n} N_{m n}(k R)\right]
\end{aligned}
$$

In this expression (2), $\mathrm{R}$ is the radius vector of the origin inside the particle, $k=2 \pi / \lambda$ is the wave number of the particle, and $\mathrm{m}_{\mathrm{c}}$ is the complex refractive index of the particle relative to the external medium. The matrices 错误!未找到引用源。and 错 误!未找到引用源。 are constructed by integrating the vector spherical wave function on the particle surface. These spherical functions are the products of famous analytic functions based on the Bessel and Wigner D functions. The scattering field expansion coefficients $\mathrm{p}$ and $\mathrm{q}$ are based on Henkel function(Hellmers et al. 2011), and the incident field expansion coefficients $\mathrm{a}$ and $\mathrm{b}$ are calculated based on the first type of Bessel function.

$$
\begin{aligned}
& M_{m n}(k R)=(-1)^{m} d_{n} h_{n}^{(\mathrm{l})}(k R) C_{m n}(\vartheta) \exp (i m \varphi) \\
& N_{m n}(k R)=(-1)^{m} d_{n}\left\{\begin{array}{c}
\frac{n(n+1)}{k R} h_{n}^{(\mathrm{l})}(k R) p_{m n}(\vartheta) \\
+\frac{1}{k R}\left[k R h_{n}^{(1)}(k R)\right] B_{m n}(\vartheta)
\end{array}\right\} \exp (\operatorname{im} \varphi)
\end{aligned}
$$

Maxwell's linear and boundary conditions determine that there must be a linear relationship between the incidence field coefficient（错误!未找到引用源。, 错误!未找到引用源。) and the scattering field coefficient（错误!未找到引用源。, 错 误!未找到引用源。). It is expressed by T-matrix :

$$
\left[\begin{array}{l}
p \\
q
\end{array}\right]=T \cdot\left[\begin{array}{l}
a \\
b
\end{array}\right]=\left[\begin{array}{ll}
T^{11} & T^{12} \\
T^{21} & T^{22}
\end{array}\right] \cdot\left[\begin{array}{l}
a \\
b
\end{array}\right]
$$

Similarly, we can write down the linear relations related to the incident field coefficient and internal field coefficient, scattering field coefficient and internal field coefficient:

$$
\begin{gathered}
{\left[\begin{array}{l}
a \\
b
\end{array}\right]=\left[\begin{array}{ll}
Q^{11} & Q^{12} \\
Q^{21} & Q^{22}
\end{array}\right] \cdot\left[\begin{array}{l}
c \\
d
\end{array}\right]} \\
{\left[\begin{array}{l}
p \\
q
\end{array}\right]=-\left[\begin{array}{ll}
\operatorname{Rg} Q^{11} & \operatorname{Rg} Q^{12} \\
\operatorname{Rg} Q^{21} & \operatorname{Rg} Q^{22}
\end{array}\right] \cdot\left[\begin{array}{l}
c \\
d
\end{array}\right]}
\end{gathered}
$$

Combined with the above, we found that:

$$
\left[\begin{array}{ll}
T^{11} & T^{12} \\
T^{21} & T^{22}
\end{array}\right]=-\left[\begin{array}{ll}
\operatorname{Rg} Q^{11} & \operatorname{Rg} Q^{12} \\
\operatorname{Rg} Q^{21} & \operatorname{Rg} Q^{22}
\end{array}\right] \cdot\left[\begin{array}{ll}
Q^{11} & Q^{12} \\
Q^{21} & Q^{22}
\end{array}\right]^{-1}
$$

$$
T=-\operatorname{Rg} Q \cdot Q^{-1}
$$

The probability density of completely randomly oriented particles in all directions is 错误! 未找到引用源。(Hovenier et al, 2000).According to the optical properties of the far-field scattering and the orthogonality of the Wigner D function( Mattis, 1981), it can be obtained that the sub-matrix 错误!未找到引用源。 of the randomly oriented non-spherical particle T-matrix are all diagonal matrix, where the asterisk represents complex conjugate, and the extinction, scattering and absorption cross sections can be calculated as follows:

$$
\begin{gathered}
C_{e x t}=-\frac{1}{k^{2}\left|E_{0}^{i n c}\right|^{2}} \operatorname{Re} \sum_{n=1}^{\infty} \sum_{m=-n}^{n}\left[a_{m n}\left(p_{m n}\right)^{*}+b_{m n}\left(q_{m n}\right)^{*}\right] \\
C_{s c a}=-\frac{1}{k^{2}\left|E_{0}^{i n c}\right|^{2}} \sum_{n=1}^{\infty} \sum_{m=-n}^{n}\left[\left|p_{m n}\right|^{2}+\left|q_{m n}\right|^{2}\right] \\
C_{a b s}=C_{e x t}-C_{s c a}
\end{gathered}
$$

When aerosol particles are randomly oriented, their average extinction, scattering and absorption cross sections are:

$$
\begin{gathered}
\left\langle C_{e x t}\right\rangle=-\frac{2 \pi}{k^{2}} \operatorname{Re} \sum_{n=1}^{\infty} \sum_{m=-n}^{n}\left[T_{m u m n}^{11}+T_{m m m n}^{22}\right] \\
\left\langle C_{s c a}\right\rangle=\frac{2 \pi}{k^{2}} \sum_{m=1}^{\infty} \sum_{m=-n}^{n} \sum_{n=1}^{\infty} \sum_{m=-n}^{n} \sum_{k=1}^{2} \sum_{l=1}^{2}\left|T_{m m m^{\prime}}^{2}\right|^{2} \\
\left\langle C_{a b s}\right\rangle=\left\langle C_{e x t}\right\rangle-\left\langle C_{s c a}\right\rangle
\end{gathered}
$$

When aerosol particles are randomly oriented, their average extinction, scattering and absorption cross sections are:

$$
\begin{aligned}
& Q_{e x t}=\frac{C_{e x t}}{G} \\
& Q_{s c a}=\frac{C_{s c a}}{G} \\
& Q_{a b s}=\frac{C_{a b s}}{G}
\end{aligned}
$$

Where $\mathrm{G}$ is the projected cross-sectional area of the scattered aerosol particle perpendicular to the direction of incident wave. For non-spherical particles, $\mathrm{G}$ can be obtained in two ways, $G=\pi \cdot r_{v}^{2}$, where 错误!未找到引用源。 is the equal volume spherical radius of aerosol particles, or 错误!未找到引用源。， where 错误!未找到引用源。 $r_{s}$ is the sphere radius of the equal surface area of the aerosol particle. The $\mathrm{T}$ matrix of randomly oriented aerosol particles can be calculated according to the $\mathrm{T}$ matrix of any orientation of particles, so it is not necessary to calculate the T matrix of all oriented particles, but only need to calculate once. 


\section{COMPARISON OF LIGHT SCATTERING CHARACTERISTICS OF SPHERICAL AND NON-SPHERICAL PARTICLE}

\subsection{Comparison of Calculated Results of Light Scattering Characteristics of Spherical Aerosol Particles by T-matrix Method and Lorenz-Mie Theory}

\begin{tabular}{|c|c|c|c|c|c|c|c|c|c|c|c|c|c|c|c|c|c|c|c|c|}
\hline & \multicolumn{2}{|c|}{$\lambda=355 \mathrm{~nm}$} & \multicolumn{2}{|c|}{$\lambda=532 \mathrm{~nm}$} & \multicolumn{2}{|c|}{$\lambda=1064 \mathrm{~nm}$} & \multicolumn{3}{|c|}{$\lambda=355 \mathrm{~nm}$} & \multicolumn{2}{|c|}{$\lambda=532 \mathrm{~nm}$} & \multicolumn{2}{|c|}{$\lambda=1064 \mathrm{~nm}$} & \multicolumn{3}{|c|}{$\lambda=355 \mathrm{~nm}$} & \multicolumn{2}{|c|}{$\lambda=532 \mathrm{~nm}$} & \multicolumn{2}{|c|}{$\lambda=1064 \mathrm{~nm}$} \\
\hline & atrix & Mie & T-Matrix & Mie & T-Matrix & Mie & & -Matrix & Mie & T-Matrix & Mie & T-Matrix & Mie & & Matrix & Mie & T-Matrix & Mie & T-Matrix & Mie \\
\hline 0.10 & 1.641344 & 1.640512 & 0.486592 & 0.486346 & 0.047296 & 0.047272 & 0.10 & 1.558299 & 1.557511 & 0.449213 & 0.448986 & 0.033642 & 0.033625 & 0.10 & 0.083045 & 0.083001 & 0.037379 & 0.037360 & 0.013654 & 0.013647 \\
\hline 0.50 & 2.694459 & 2.693095 & 2.421873 & 2.420650 & 3.642561 & 3.640722 & 0.50 & 2.283019 & 2.281864 & 2.132166 & 2.131084 & 3.503898 & 3.502125 & 0.50 & 440 & 231 & 0.289707 & 0.289566 & 663 & 97 \\
\hline 1.00 & 2.148971 & 2.148268 & 2.241089 & 2.239953 & 2.421869 & 2.420650 & 1.00 & 1.5 & 1.58 & 583 & 1.75 & 2.1 & 2.1 & 1. & 08 & 0.55 & 506 & 60 & 10 & 9566 \\
\hline 1.50 & 2.321925 & 2.320760 & 150050 & 49331 & 2.708549 & 2.7071 & 1.5 & 1.643878 & 1.643049 & 1.586667 & 86073 & 2.295556 & 395 & 1.5 & 0.678047 & 0.677711 & 383 & 0.563258 & 2993 & 12788 \\
\hline 2.00 & 2.113225 & 2.112184 & 2.130111 & 2.129052 & 2.241091 & 2.239953 & 2.00 & 033 & 1.352347 & 798 & 1.488046 & 1.756584 & 1.755693 & 2.00 & 192 & 0.759837 & 0.641313 & 0.641006 & 0.484507 & 0.484260 \\
\hline 2.50 & 2.208117 & 2.207048 & 2.123557 & 2.122687 & 2.506329 & 2.505060 & 2.50 & 1.396311 & 1.395611 & 1.412576 & 1.411968 & 1.965575 & 1.964581 & 2.50 & 0.811806 & 0.811437 & 0.710981 & 0.710719 & 0.540754 & 0.540479 \\
\hline 3.00 & 2.106978 & 2.105966 & 2.114271 & 2.113218 & 2.150046 & 2.149331 & 3.00 & 1.252778 & 1.252171 & 1.353054 & 1.351269 & 1.586670 & 1.586073 & 3.00 & 0.854200 & 0.853795 & 0.761217 & 0.760849 & 0.563376 & 0.563258 \\
\hline
\end{tabular}

Table 1. Calculation results of extinction, scattering and absorption efficiency factors of spherical particles using T-matrix method and Lorenz-Mie theory varying with particle size at different wavelengths

In Table 1, the extinction efficiency factors, scattering efficiency factors and absorption efficiency factors of spherical particles with a refractive index of $1.55+0.01 \mathrm{i}$ were calculated by T-Matrix method and Lorenz-Mie theory respectively, where the particle radius varied from $0.1 \mathrm{~nm}$ to $3 \mathrm{~nm}$.As can be seen from the above table, with the increase of particle size, the light scattering results calculated by T-matrix and Lorenz-Mie theory at different wavelengths differ little. Taking extinction efficiency factor as an example, the relative error of the two methods at $532 \mathrm{~nm}$ is only 错误!未找到引用源。, no matter how the wavelength and particle size change, the dispersive characteristics of T-Matrix method and Lorenz-Mie theory in calculating spherical aerosol particles are completely consistent, which proves that the correctness and reliability of the T-matrix method in simulating the scattering of spherical particles, and verifies the high accuracy of the calculation results of the Tmatrix method.

\subsection{Spherical and Non-spherical Aerosol Particles at Different Particle Size}
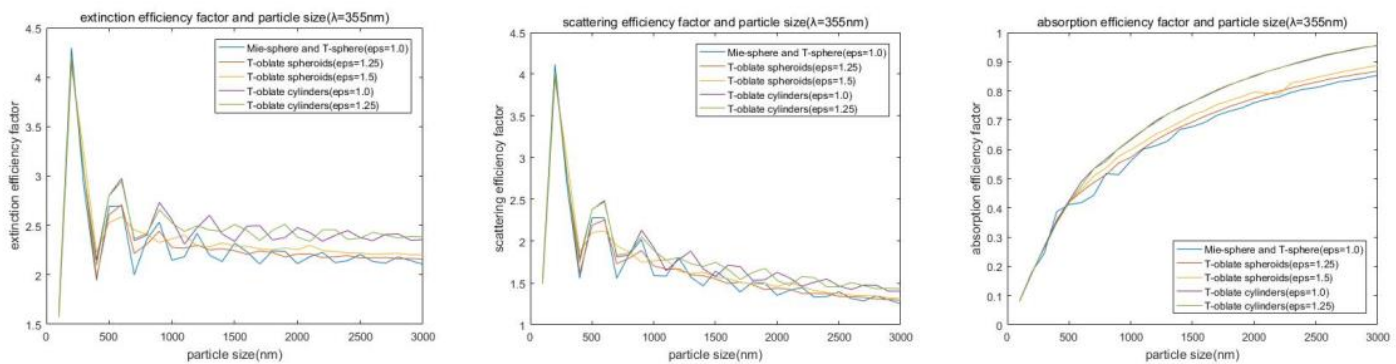

Figure 3. The extinction efficiency factor (left), scattering efficiency factor (middle) and absorption efficiency factor (right) of sphere, oblate sphere and oblate cylinder under the diameter $0 \sim 3 \mathrm{um}$ were compared by T-matrix method and Lorenz-Mie theory (The wavelength was $355 \mathrm{~nm}$ and the refractive index was $1.55+0.01 \mathrm{i}$ )
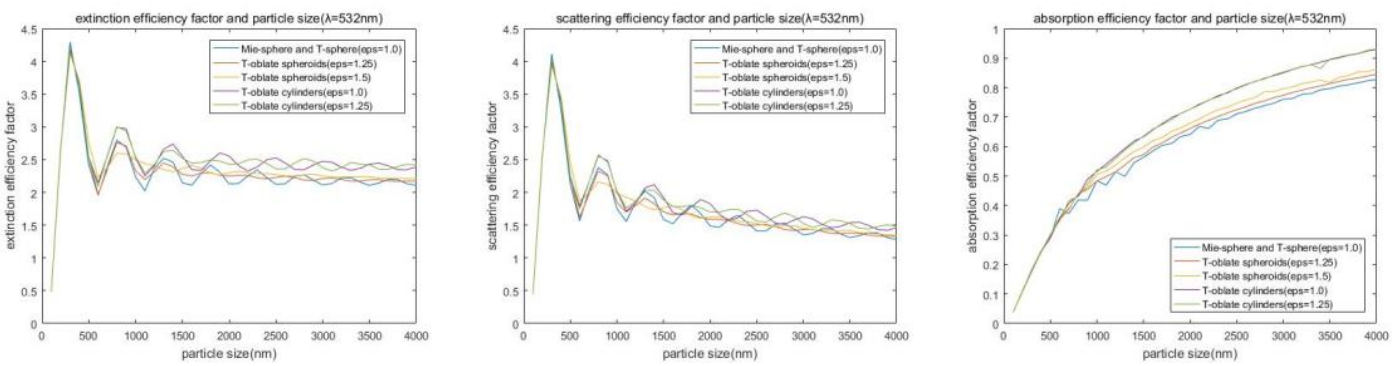

Figure 4. The extinction efficiency factor (left), scattering efficiency factor (middle) and absorption efficiency factor (right) of sphere, oblate sphere and oblate cylinder under the diameter $0 \sim 4$ um were compared by T-Matrix method and Lorenz-Mie theory(The wavelength was $532 \mathrm{~nm}$ and the refractive index was $1.55+0.01 \mathrm{i}$ ) 

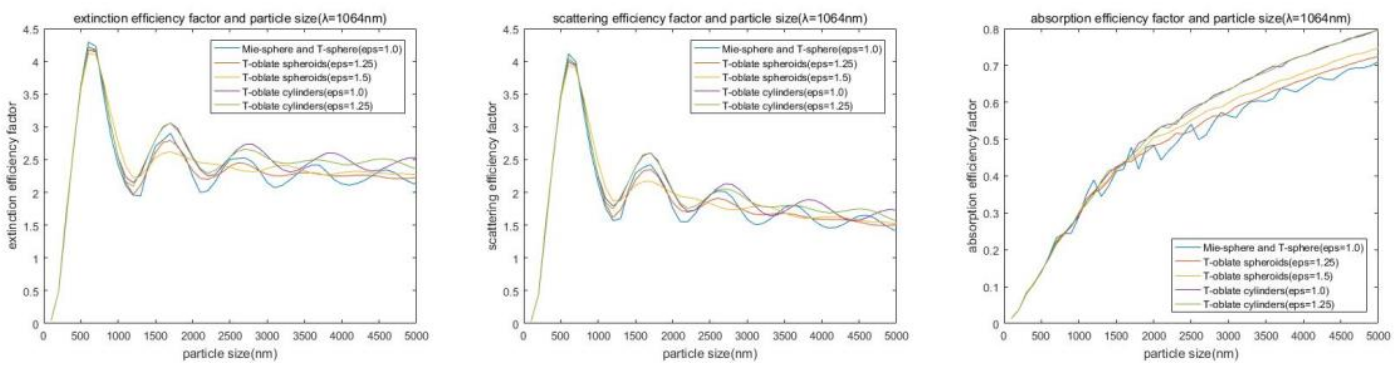

Figure 5. The extinction efficiency factor (left), scattering efficiency factor (middle) and absorption efficiency factor (right) of sphere, oblate sphere and oblate cylinder under the diameter $0 \sim 5$ um were compared by T-matrix method and Lorenz-Mie theory (The wavelength was $1064 \mathrm{~nm}$ and the refractive index was $1.55+0.01 \mathrm{i}$ )

Figure 3, 4 and 5 simulate the light scattering characteristics of aerosol particles with different shapes under different particle sizes at wavelength $355 \mathrm{~nm}, 532 \mathrm{~nm}$ and $1064 \mathrm{~nm}$. As can be seen from the figures, when the particle size of the aerosol is smaller than the wavelength, its extinction efficiency and scattering efficiency factor will have a peak value, and show a normal distribution in the region. Then, when the particle size of the aerosol is larger than the wavelength, its extinction efficiency and scattering efficiency factor will oscillate and eventually tend to a constant value. In the case of particle size $0 \sim 5 \mathrm{um}$, the absorption efficiency factors of three wavelengths of aerosol particles increased infinitely to 0.8 , and the increase rate gradually slowed down with the particle size increasing. The extinction efficiency and scattering efficiency factor of different shapes are very similar, and the extinction scattering ability of cylindrical particles is stronger than spherical particles. In TMatrix method to calculate different particle sizes of spherical aerosol particles, regardless of the aerosol particles are spheroids, cylinder, its light scattering properties trend is consistent, it is illustrates that at same the wavelength, the spherical or non-spherical particles had no effect on light scattering properties. It is proved that using T-Matrix method to calculate the light scattering properties of the non-spherical aerosol particles is reliable.

\subsection{Spherical and Non-spherical Aerosol Particles at Different Wavelengths}
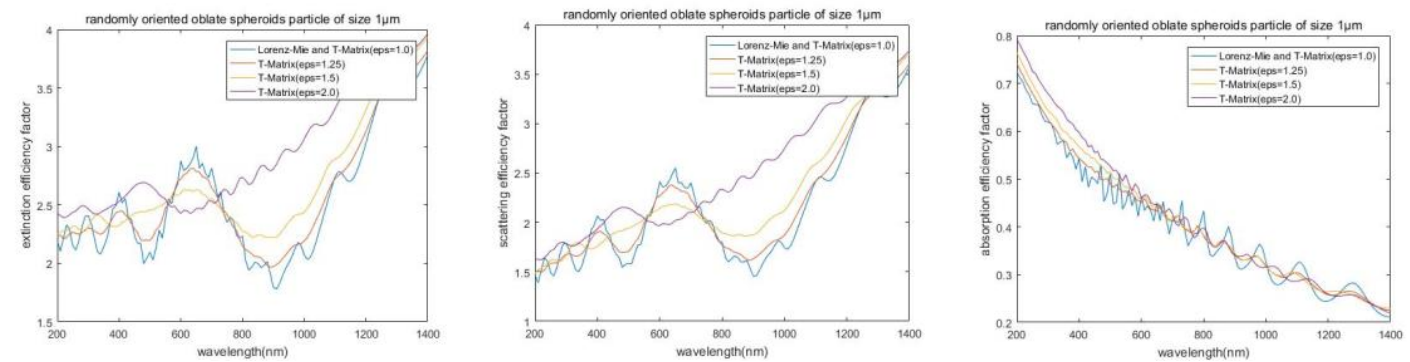

Figure 6. Comparison of extinction efficiency factor (left), scattering efficiency factor (middle) and absorption efficiency factor (right) of randomly oriented spheroids by T-matrix method and Lorenz-Mie theory with different wavelengths and shapes (refractive index is $1.55+0.01 \mathrm{i}$, particle size is 1 micron)
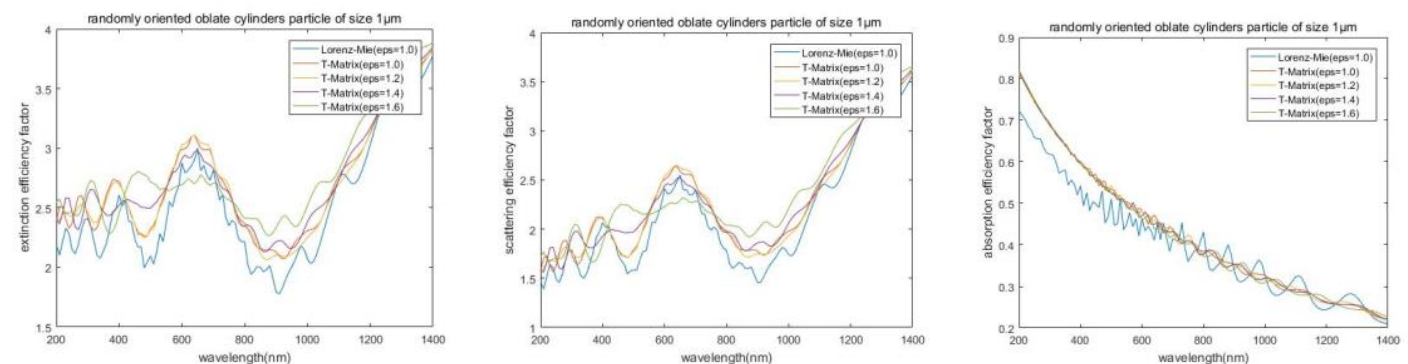

Figure 7. Comparison of extinction efficiency factor (left), scattering efficiency factor (middle) and absorption efficiency factor (right) of randomly oriented cylinders by T-matrix method and Lorenz-Mie theory with different wavelengths and shapes (refractive index is $1.55+0.01 \mathrm{i}$, particle size is 1 micron)

It can be seen from figure 6 and 7 that in the surrounding medium with sand and dust (refractive index was $1.55+0.01 \mathrm{i}$ ), the particle size is $1 \mu \mathrm{m}$, either with $\mathrm{T}$ - matrix method or Lorenz Mie theory to calculate the light scattering properties of aerosol particles, with the increase of the incident wavelength, the extinction efficiency factor and the scattering efficiency factor of the oblate cylinder whose longitudinal resonance peak wavelength is concentrated in the $600 \mathrm{~nm}$ to $800 \mathrm{~nm}$. there is a continuous oscillation in less than $1000 \mathrm{~nm}$. With the change of the shape of the non- spherical particles, the extinction 
efficiency factor and scattering efficiency factor whose longitudinal resonance wavelength moves to the right, but the peak remains at $600 \mathrm{~nm}$ to $800 \mathrm{~nm}$. When the wavelength is greater than $1000 \mathrm{~nm}$, two kinds of method to calculate the extinction efficiency factor and the scattering efficiency factor increases sharply and tends to consistent. It is shows that $\mathrm{T}$ Matrix method to calculate the extinction efficiency of spherical particles is wavelength independent. In short, oblate cylinders particle and oblate spheroids particle have similar extinction scattering properties. The absorption efficiency factor of spherical aerosol particles calculated by Lorenz-Mie theory tends to 0.2 with the increase of incident light wave length, while the absorption efficiency factor of non-spherical and spherical aerosol particles calculated by T-matrix method finally tends to 0.3 . It is indicated that there are differences in the two methods involved in the calculation. The reason for the difference between the scattering characteristics of the LorenzMie theory and the T-matrix method is related to the shape of the particles. This is owing to the cross-sectional area of nonspherical aerosol particles perpendicular to the direction of incident wave is irregular. It is slightly different from that of spherical aerosol particles under ideal conditions, which leads to this situation.

As shown in the above figure, it can be seen that using two methods to calculate the light scattering properties of spherical and non-spherical aerosol particles have the same general trend. But there are some differences between the calculation accuracy. When using the T-Matrix method, it can be known that the calculation result is independent of the wavelength of the incident light. It is only related to its inherent physical properties. Therefore, it can be proved that the T-Matrix method is correct in calculating non-spherical aerosol particles, and the advantage is that it can calculate the subtle difference between aerosol particles of the same kind and different nonspherical degrees, so it has higher precision than the LorenzMie theory.

\section{CONCLUSION}

In order to understand the reliability of the T-matrix method for calculating non-spherical aerosol particles, the T-Matrix method and Lorenz-Mie theory are used to calculate the light scattering properties of spherical aerosol particles at the same shape and different wavelengths. It can be seen that it is the same using TMatrix method and the Lorenz Mie theory to calculate the scattering characteristics of spherical particles. When calculating the scattering properties of non-spherical aerosol particles, the T-Matrix method is more advantageous than the Lorenz-Mie theory. The Lorenz-Mie theory only obtains the scattering characteristics of spherical particles accurately. For non-spherical aerosols particles with different sizes and different shapes, T-Matrix method can get the subtle difference between them more accurately and quickly, and lay a foundation for more accurate simulation of the microphysical properties of aerosol particles in the real atmosphere.

\section{ACKNOWLEDGEMENTS}

This research was funded by the National Natural Science Foundation of China (Grant No. 61865001) and the Ningxia Natural Science Foundation (Grant No. 2018AAC03103).

\section{REFERENCES}

Choi, M.K., Liebman, L.A., Brock, J.R., 1996. Finite element solution of the maxwell equations for absorption and scattering of electromagnetic radiation by a coated dielectric particle. Chemical Engineering Communications, 151(1), 5-17. 10.1080/00986449608936538

Draine, B.T., 1988. The discrete-dipole approximation and its application to interstellar graphite grains. The Astrophysical Journal, 333(2),848-872. 10.1086/166795

Farafonov, Victor, G., Ilin, Vladimir, 2005. Light scattering by nonspherical particles: some theoretical aspects. Proceedings of SPIE - The International Society for Optical Engineering,5829, 109-116. 10.1117/12.617257

Hoyningen-Huene, W.V., Posse, P., 1997. Nonsphericity of aerosol particles and their contribution to radiative forcing. Journal of Quantitative Spectroscopy and Radiative Transfer,57(5), 651-668. 10.1016/s0022-4073(96)00153-7

Hovenier, M.I., Mishchenko, J.W., Travis, L.D., 2000. Light scattering by nonspherical particles: theory, measurements, and applications. 10.1088/0957-0233/11/12/705.

Huber, E., Frost, M., 1998. Light scattering by small particles. Journal of Water Supply: Research and Technology-AQUA Online,47(2),87-94. 10.2166/aqua.1998.14

Hellmers, J., Schmidt, V., Wriedt, T., 2011. Improving the numerical stability of t-matrix light scattering calculations for extreme particle shapes using the nullfield method with discrete sources. Journal of Quantitative Spectroscopy and Radiative Transfer, 112(11), 1679-1686. 10.1016/j.jqsrt.2011.02.004

Jin, J.,2002. The finite element method in electromagnetics. Journal of the Japan Society of Applied Electromagnetics, 1,39-40. doi:http://dx.doi.org/

Mishchenko, M.L., Travis, L. D., Lacis, A.A, 2002. Book review: scattering, absorption, and emission of light by small particles / cambridge university press. Space Science Reviews, 442 .

Mur, G.,1981. Absorbing boundary conditions for the finitedifference approximation of the time-domain electromagneticfield equations. IEEE Transactions on Electromagnetic Compatibility, EMC-23(4),377-382.

10.1109/TEMC.1981.303970

Mishchenko, M.I., Travis, L.D., 1994. T-matrix computations of light scattering by large spheroidal particles. Optics Communications, 109(1-2), 16-21. 10.1016/00304018(94)90731-5

Mishchenko, M.I., Travis, L.D., Macke, A., 2000. T-matrix method and its applications. 10.1016/B978-0124986602/50033-1.

Mattis, D.C., 1981. Quantum theory of angular momentum. 10.1007/978-3-642-83238-3_3.

Park, Y., Minh, Y., 2012. Journal of quantitative spectroscopy and radiative transfer. Journal of Quantitative Spectroscopy and Radiative,Transfer, urn: issn:0022-4073.

Purcell, E.M., Pennypacker, C.R., 1973. Scattering and absorption of light by nonspherical dielectric grains. The 


\section{Astrophysical Journal, 186,705. 10.1086/152538}

Rother, T., 1998. Generalization of the separation of variables method for non-spherical scattering on dielectric objects. Journal of Quantitative Spectroscopy and Radiative Transfer, 60(3), 335-353. 10.1016/s0022-4073(98)00010-7

Schneider, J.B., 2004. Plane waves in fdtd simulations and a nearly perfect total-field/scattered-field boundary. IEEE Transactions on Antennas and Propagation, 52(12), 3280-3287. 10.1109/tap.2004.836403

Schmidt, K., Wauer, J., Rother, T., 2003. Application of the separation of variables method to plane wave scattering on nonaxisymmetric particles. Lidar Multiple Scattering Experiments. International Society for Optics and Photonics, $10.1117 / 12.512338$.

Spurr, R., Wang, J., Zeng, J., Mishchenko, M.I., 2012. Linearized t-matrix and mie scattering computations. Journal of Quantitative Spectroscopy \& Radiative Transfer, 113(6),425-

\section{10.1016/j.jqsrt.2011.11.014}

Umashankar, K., Taflove, A., 1982. A novel method to analyze electromagnetic scattering of complex objects.IEEE Transcations on Electromagnetic Compatibility, EMC24(4),397-405. 10.1109/temc.1982.304054

Wielaard, D.J., Mishchenko, M.I., Macke, A., Carlson, B.E., 1997. Improved t-matrix computations for large, nonabsorbing and weakly absorbing nonspherical particles and comparison with geometrical-optics approximation. Applied Optics, 36(18), 4305-4313. 10.1364/AO.36.004305

Waterman, P. C., 1965. Matrix formulation of electromagnetic scattering. Proceedings of the IEEE, 53(8), 805-812. 10.1109/PROC.1965.4058

Yang, P., Liou, K.N., 1996. Finite-difference time domain method for light scattering by small ice crystals in threedimensional space. Journal of the Optical Society of America A 13(10), 2072-2085. 10.1364/JOSAA.13.002072 